punto org

Collana diretta da Luigi Maria Sicca

10 
ROSARIO DiANA

\title{
DISAPPARTENENZA DELL'IO
}

\section{Filosofia e musica verso Samuel Beckett}

\author{
LIBRO-CON-DISCO
}

musiche di

Giancarlo Turaccio, Lorenzo Pone, Chiara Mallozzi

Bernardo Maria Sannino, Rosalba Quindici, Salvatore Carannante

\author{
prefazione \\ Luigi Maria Sicca
}

Editoriale Scientifica

Napoli 
La pubblicazione di questo lavoro lavoro è stata resa possibile grazie ad un finanziamento dell'Istituto per la Storia del Pensiero Filosofico e Scientifico Moderno (ISPF) del Consiglio Nazionale delle Ricerche - Fondi Miur Prin 2009.

Tutti i diritti sono riservati

(C) 2016 Editoriale Scientifica srl

Via San Biagio dei Librai 39

80138 Napoli

www.editorialescientifica.com

info@editorialescientifica.com

ISBN 978-88-6342-885-8 


\section{Indice}

7 DISAPPARTENENZA DELL'IO: BREVE STORIA DI UN LIBRO-CON-DISCO Luigi Maria Sicca

13 Introduzione

LE RAGIONI DI UN LIBRO-CON-DISCO

33 Ringraziamenti

35 DISAPPARTENENZA DELL'IO.

FilOSOFIA VERSO SAMUEl BECKETT

37 DEPOTENZIAMENTO DEL COGITO CARTESIANO

E “DISAPPARTENENZA” DELL'IO.

IN MARGINE AL DE ANTIQUISSIMA Di GIAMBATTISTA VICO

51 BORIA E TRACOLLO DELL'IO.

BECKETT E GEULINCX

83 L'OCCHIO ONTOPOIETICO.

BECKETT E BERKELEY

105 UNA LETTURA DI FINALE DI PARTITA

121 LE CICATRICI DELL'IO.

UNA TRAIETTORIA DA KRAPP A IMPROVVISO DELL'OHIO

143 Appendice

145 Musica COME FILOSOFIA.

SUONO, ASCOLTO, SPAZIO

Giancarlo Turaccio

165 Abstract 
167 Partiture

169 ARCHEA

Giancarlo Turaccio

181 KHARMAN PER SAMUEl BeCKetT

Lorenzo Pone

211 E_SITI

Chiara Mallozzi

235 PRELUdiO E OMBRA

Bernardo Maria Sannino

265 PERCIPI

Rosalba Quindici

289 PASSAGGI DI STATO

Salvatore Carannante

307 Indice dei nomi

313 Hanno scritto nella Collana punto org 


\section{Disappartenenza dell'Io: breve storia di un libro-con-disco}

Luigi Maria Sicca

Disappartenenza dell'Io vede la luce dopo una lunga gestazione: azione organizzativa densa di senso.

Nel 2012 Rosario Diana propose un tema ambizioso, da sviluppare in seno alla progetto puntOorg, a partire da alcune idee che, in dialogo con Beckett, gli consentivano di attraversare un aspetto specifico della condizione ordinaria dell'Io, quale riferimento imprescindibile del nostro dire noi stessi. Suo intento era di coinvolgere compositori impegnati nella musica di ricerca e invitarli a tradurre i nuclei concettuali del suo lavoro in un progetto compositivo. All'epoca alcuni saggi inclusi in questo volume erano già stati pubblicati. Si trattava di uno scritto su Vico, teso a mettere a punto il concetto di disappartenenza dell'Io con apertura, nelle conclusioni, all'opera di Beckett. Un secondo su Beckett e Berkeley in rapporto a Film. Un terzo era un'interpretazione di Finale di partita.

\section{$* * *$}

Libro-con-disco è il primo - nel suo genere - della nostra Collana. Rispetto ai precedenti numeri, ci troviamo di fronte a un'innovazione, sia di prodotto, sia di processo, nella misura in cui l'oggetto che il lettore ha tra le mani è esso stesso, nella sua fisicità, al contempo, sia strumento sia fine della ricerca. Sia causa che effetto di un percorso di apprendimento. Un percorso fatto di arte, in particolare di musica, in connessione alla filosofia: filosofia e musica verso Samuel Beckett, come recita il sottoti- 
tolo. Stiamo per confrontarci, come argomenterò brevemente, con un artefatto. A partire da due domande:

1. cosa è un artefatto con riferimento a questa, come ad altre esperienze di ricerca maturate in seno a puntOorg?

2. perché libro-con-disco, nel suo essere artefatto, è il perno intorno a cui ruota un'esperienza, in certo senso inedita in termini di organization studies?

Risponderò alle due domande, rammentando che artefatto è parola composta da arte e factus, fatto con arte. Dunque effetto dell'arte. Arte è innanzitutto uno stato della mente che incontra i mezzi (da $\tau \hat{\varepsilon} \chi \vee \eta)$, allo scopo di realizzare azioni che nella loro concretezza generano altre azioni. Fatto è dunque ciò che risulta dalla messa in opera di ciò che chiamiamo arte, perché idoneo a cambiare la percezione delle cose e la loro rappresentazione. Gli artefatti sono dunque oggetti latu sensu. Dicono molto delle caratteristiche degli attori organizzativi e dei loro modi di agire. Negli studi organizzativi gli artefatti, essendo espressione di un modo, sono strumento, ma anche progetto e, di conseguenza, cambiamento interiore, attraverso una relazione con i cinque sensi ${ }^{1}$. Da cui la costruzione di senso (sensemaking) e l'equivalenza tra processi mentali e sociali dell'organizzare (organizing) e processi cognitivi ${ }^{2}$. Gli artefatti possono incidere, insomma, su quel che gli economisti chiamano decision making, attraverso l'impatto sul sistema sensoriale.

È questa, secondo me, una chiave di lettura dell'esperienza del gruppo di ricerca che ha realizzato il progetto Disappartenenza dell'Io: Rosario Diana si è affidato ad attori organizzativi, per creare senso nello sviluppo della sua proposta. Si è affidato, in particolare, alla potenzialità deliberante propria degli artisti, a dei

${ }^{1}$ Cfr. P. Gagliardi, Il gusto dell'organizzazione. Estetica, conoscenza, management, Milano, Guerini \& Associati, 2011.

${ }^{2}$ Cfr. K.E. Weick, Cognitive Processes in Organizations, in B.M. Staw, (Ed.), Research in Organizational Behaviour, Greenwich Connecticut, JAI Press, 1979. 
musicisti ${ }^{3}$, che hanno saputo raccogliere la sfida lanciata, senza cedere alla tentazione di imitare o emulare semplicemente il modello concettuale proposto. Al contrario, a partire da quest'ultimo, hanno scelto anche di sperimentare proprie direzioni di indagine; dunque di pro-vocare - con i loro linguaggi e la loro grammatica fatta di scrittura (composizione) e di lettura (interpretazione) - risultati inediti, sintetizzati nella forma del librocon-disco: da studiare, da ascoltare. La potenzialità deliberante di compositori ed esecutori, percepibile e udibile, ora nel disco, è stata realizzata nel corso di un laboratorio coordinato e concertato da Giancarlo Turaccio, durante il quale sono state effettuate le registrazioni live, presso l'Università degli Studi di Napoli Federico II nei giorni di 4, 5 e 6 giugno 2013, nella Chiesa di SS. Marcellino e Festo, in collaborazione con l'Associazione Alessandro Scarlatti - Ente Morale, con l'autorevole partecipazione del clarinettista Guido Arbonelli, che nei giorni immediatamente precedenti aveva tenuto una Masterclass, organizzata dal Conservatorio Statale di Musica di Salerno "G. Martucci", sulle prassi esecutive e interpretative del clarinetto nella musica contemporanea.

L'artefatto libro-con-disco è stato il perno di questa esperienza di ricerca proprio perché, sin dall'inizio, ha svolto il ruolo di terzo pirandelliano: assente e presente, mezzo, strumento, in quanto tale, per definizione, non neutrale rispetto allo scopo. È stato così nell'esperienza del gruppo di compositori-ricercatori che hanno collaborato a questo progetto. Prima ancora che lo stesso libro-con-disco, di fatto, prendesse la forma che oggi possiamo maneggiare, osservare, leggere ed ascoltare, esso ha condizionato le idee e l'evoluzione dell'intero percorso compiuto dalla ricerca, generando interessanti e fruttuose interazioni dialogiche e dialettiche fra i vari momenti diacronici dell'indagine in cammino.

${ }^{3}$ L.M. Sicca (a cura di), I linguaggi dell'organizzare. Musica e testo tra dono e disinteresse, Napoli, Editoriale Scientifica, 2013, p. 17. 
Dalla metà di luglio 2012 il gruppo di compositori-ricercatori, oggi co-protagonisti di questa pubblicazione, avviò un ciclo di incontri seminariali mensili teso ad una progressiva esplorazione delle possibili direzioni di lavoro tra musica e filosofia, sulla linea di un ideale confine fra le due. Una linea talvolta incerta, da percorrere con attenzione e prudenza, come raccomandano l'introduzione di Rosario Diana - che illustra e argomenta l'impianto teorico dell'intera operazione - e il saggio di Giancarlo Turaccio a essa collegato, che tematizza il punto di vista di chi compone musica.

Si consolidava uno dei tratti caratterizzanti l'identità di molti dei progetti puntOorg: il lavoro di artisti (in questo caso musicisti-ricercatori) e di "scienziati" (in questo caso un filosofo) compiuto non tanto con lo sguardo rivolto esclusivamente ai propri ambiti specifici (rispettivamente la musica e la filosofia), ma svolto con l'atteggiamento di chi porta acqua per alimentare uno sviluppo più consapevole nella riflessione su possibili modi di "stare insieme". Insomma, il filosofo e il musicista - depositari di saperi che dalla ricerca di base, attraverso tecnica e tecnologia, sanno tradursi in produttività, fino ad assurgere a "senso comune", secondo la tradizionale struttura delle rivoluzioni scientifiche ${ }^{4}$ - interagiscono performativamente: un modo di operare assai lontano quindi dall'idea di intrattenimento ${ }^{5}-$ spesso associato alla musica - e di inutilità - talvolta ingenua-

${ }^{4}$ Cfr. T.S. Kuhn, La struttura delle rivoluzioni scientifiche (1962), tr. it. di A. Carugo, Torino, Einaudi, 1978.

${ }^{5}$ Un'idea, questa, con non poche ripercussioni in termini di formazione e di politica universitaria, in Italia ancora assai lontana dalla tradizione mitteleuropea. Basti pensare a come la riforma dell'istruzione musicale ha trasformato sulla carta, in seno al MIUR, le istituzioni scolastiche (attraverso l'AFAM), senza di fatto definire un piano di interventi micro-organizzativi per incidere nel profondo dei processi di formazione dei formatori. 
mente rinfacciata alla filosofia. Filosofia e musica, già tematizzate da noi di puntOorg, come "fonti di sapere manageriale"6, alla cui sorgente risiedono risorse (che sono anche archetipi) non scarse (per esempio il suono che si fa musica), adeguate ad aprire ulteriori piste di pensiero e di azione economico-organizzativa.

In tal senso, libro-con-disco si iscrive nella sfida, lanciata dalla rete di ricerca internazionale puntOorg, di cercare nuove domande e possibili risposte a un modo di stare insieme, caratterizzato da intervalli asintotici tra momenti di espansione e periodi di crisi sempre più lunghi. Partecipando, così, a quell'amplissima riflessione teorica - che attraversa oggi a livello internazionale tutti i contesti accademici che si interessano di humanities - sul linguaggio, inteso nella sua potente trasversalità, rispetto alle numerose tematizzazioni che ciascuno di noi può mettere in campo nel lavoro esclusivamente intradisciplinare.

$$
* * *
$$

Questo libro è il risultato di un'intensa e felice cooperazione tra persone e istituzioni, ciascuna delle quali ha favorito la circolazione di punti di vista tra i ricercatori coinvolti: 1'Università degli Studi di Napoli Federico II; l'Istituto per la Storia del Pensiero Filosofico e Scientifico Moderno (ISPF) del Consiglio Nazionale delle Ricerche; il Conservatorio di Musica di Napoli San Pietro a Majella; il Conservatorio di Musica di Salerno G. Martucci; l'Associazione Alessandro Scarlatti - Ente Morale; la Federazione Cemat.

${ }^{6}$ L.M. Sicca, Alla fonte dei saperi manageriali. Il ruolo della musica nella ricerca per l'innovazione e per la formazione delle risorse umane, Napoli, Editoriale Scientifica, 2012, elaborazione in forma scritta della Prolusione per l'inaugurazione dell'A.A. 2011-2012 che tenni, con il titolo E per questo preferisci il dispari, presso il Conservatorio di Musica di Benevento N. Sala. 
Il disegno di copertina è l'esito della collaborazione con il piccolo, poetico Michelangelo: recuperare il gesto infantile è, da sempre, un tratto distintivo della Collana. Naturale contrappeso a dibattiti complessi, densi, adulti, sempre sensibili allo stupore che può derivare dall'innovazione e dalle sperimentazioni nelle pratiche del pensiero. Verso la costruzione del linguaggio.

Napoli, 15 dicembre 2013 